\title{
The role of surgical expertise with regard to chronic postoperative inguinal pain (CPIP) after Lichtenstein correction of inguinal hernia: a systematic review
}

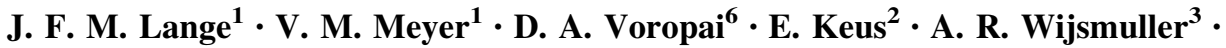 \\ R. J. Ploeg ${ }^{4}$ J. P. E. N. Pierie ${ }^{5}$
}

Received: 16 July 2015/ Accepted: 16 March 2016/Published online: 5 April 2016

(c) The Author(s) 2016. This article is published with open access at Springerlink.com

\begin{abstract}
Objective The aim of this study was to evaluate whether a relation exists between surgical expertise and incidence of chronic postoperative inguinal pain (CPIP) after inguinal hernia repair using the Lichtenstein procedure .

Background CPIP after inguinal hernia repair remains a major clinical problem despite many efforts to address this problem. Recently, case volume and specialisation have been found correlated to significant improvement of outcomes in other fields of surgery; to date these important factors have not been reviewed extensively enough in the context of inguinal hernia surgery.

Methods A systematic literature review was performed to identify randomised controlled trials reporting on the incidence of CPIP after the Lichtenstein procedure and including the expertise of the surgeon. Surgical expertise was subdivided into expert and non-expert.

Results In a total of 16 studies 3086 Lichtenstein procedures were included. In the expert group the incidence of
\end{abstract}

J. F. M. Lange

jfmlange@hotmail.com; j.lange@umcg.nl

1 Department of Surgery, University Medical Center Groningen, Groningen, The Netherlands

2 Department of Critical Care, University Medical Center Groningen, Groningen, The Netherlands

3 IRCAD/EITS, Department of General, Digestive and Endocrine Surgery, University Hospital of Strasbourg, Strasbourg, France

4 Nuffield Department of Surgical Sciences, University of Oxford, Oxford, UK

5 Postgraduate School of Medicine, University Groningen, Groningen, The Netherlands

6 St Jansdal Hospital, Harderwijk, The Netherlands
CPIP varied between 6.9 and $11.7 \%$ versus an incidence of 18.1 and $39.4 \%$ in the non-expert group. Due to the heterogeneity between groups no statistical significance could be demonstrated.

Conclusion The results of this evaluation suggest that an association between surgical expertise and CPIP is highly likely warranting further analysis in a prospectively designed study.

Keywords Lichtenstein - Chronic pain - Inguinal pain . Expert $\cdot$ Systematic review $\cdot$ Inguinal hernia

\section{Introduction}

Inguinal hernia has a lifetime occurrence risk of $20 \%$ in men, making its surgical repair one of the most performed surgical procedures worldwide. Annually in the Netherlands over 20.000 inguinal hernias are surgically corrected. In the past few decades the quality of inguinal hernia surgery has improved drastically. Recurrence rates have fallen below $5 \%$ with the introduction of mesh repair and patients are treated in a day setting. Despite these achievements, inguinal hernia repair remains to be associated with one major complication: chronic postoperative inguinal pain (CPIP). Up to $10-20 \%$ of patients report CPIP, defined as chronic inguinal pain more than 3 months after surgery. Inguinal hernia surgeons have developed various techniques to address this problem, but CPIP remains a major issue to this day.

There is strong evidence that the surgeon's case volume and specialisation will improve outcome of major surgical procedures, i.e. gastrectomy, esophagectomy, pancreatic surgery and rectal cancer surgery [1,2]. This may also apply to the complication of CPIP in inguinal hernia surgery. 
In many Western countries nowadays the Lichtenstein technique is gold standard. In the majority of patients, this technique is performed by residents in an early phase of their training. Also surgeons not specialised in hernia surgery perform this operation albeit in low numbers. The role of case volume and specialisation in more common surgeries including inguinal herniaplasties has not clearly been defined yet. Expertise might especially affect the outcome of CPIP.

We compared the results of expert surgeons versus nonexpert surgeons ascertaining any possible correlation between expertise and CPIP after inguinal hernia surgery. The objective of this study was to conduct a systematic review of incidence of CPIP after the Lichtenstein procedure with surgeons' expertise as the main variable.

\section{Materials and methods}

\section{Search strategy}

Data collection and analysis were performed according to the Preferred Reporting Items for Systematic Reviews and Meta-Analyses (PRISMA) statement [3]. The following databases were searched: CENTRAL on The Cochrane Library, The National Library of Medicine (MEDLINE/ PubMed) and The Intelligent Gateway to Biomedical and Pharmacological Information (EMBASE) for randomised trials. The search strategy, date and time span of search are provided in Table 1. We chose a search interval of the past 10 years to obtain and analyse the most recent data. In addition, a cross-reference search of relevant trials was performed.

\section{Selection of studies}

Only prospective randomised controlled trials were included. In the selected studies the Lichtenstein technique with its variations (including all types of glue, staples, stitches, fibrin or any type of mesh) had to be addressed, reporting on CPIP (inguinal pain more than 3 months after surgery).

The inclusion was restricted to studies with focus on primary inguinal hernia repair of uni- or bilateral hernias, irrespective of language, size and blinding. Studies had to describe whether an expert, non-expert or resident performed the procedure. An expert surgeon was defined as one who performed more than 150 Lichtenstein procedures each year or alternatively, or as one who was dedicated to abdominal wall surgery. In case of any doubt or missing data, the corresponding author could be contacted. Exclusion criteria were case series and retrospective studies, studies in children, studies evaluating hernia repair techniques other than the Lichtenstein procedure and studies on recurrent hernias.

\section{Outcome}

Primary end point was occurrence of CPIP. CPIP was defined as an awareness of chronic pain lasting longer than 3 months after hernia repair. There were no other outcomes. CPIP was subdivided into four time intervals at 3 , 6, 12 and 24 months.

\section{Data}

Initial screening of all hits and selection based on title and abstract was performed by one author (JL or VM). All selected abstracts were evaluated based on full text by three independent reviewers (JL, DV and VM). In case of disagreement, consensus was reached through discussion. In case of any overlap of patients between reported series, only the largest cohort was included. All data were extracted into a standard data form including author, journal, year of publication, study type, number of patients, number of Lichtenstein procedures, incidence of CPIP after $3,6,12$ or 24 months, level of expertise of operating surgeons (dedicated/expert, non expert, resident) and corresponding author, stated outcome and duration of follow-up period.

\section{Data analysis and bias detection}

We compared the incidence of CPIP between studies reporting on hernia surgeons (expert group) and surgeons with no special attention for hernia repair/residents (non

Table 1 Search strategy in Pubmed, EMBASE and Cochrane Library

\begin{tabular}{|c|c|c|c|}
\hline Databases & Search strategy & Date of search & Time span \\
\hline Pubmed & $\begin{array}{l}\text { "Lichtenstein" AND/OR "inguinal hernia" AND "chronic pain" } \\
\text { AND/OR "CPIP" }\end{array}$ & 20-11-2015 & 2006 - date of search \\
\hline EMBASE & $\begin{array}{l}\text { "Lichtenstein" AND/OR “inguinal hernia" AND “chronic pain" } \\
\text { AND/OR "CPIP" }\end{array}$ & 20-11-2015 & 2006-date of search \\
\hline Central (Cochrane Library) & $\begin{array}{l}\text { "Lichtenstein" AND/OR "inguinal hernia" AND "chronic pain" } \\
\text { AND/OR "CPIP" in cohort studies and randomised controlled trials }\end{array}$ & 20-11-2015 & 2006-date of search \\
\hline
\end{tabular}


expert group). Qualification of biases in randomised controlled trials was performed according to the Cochrane handbook of systematic reviews with the Cochrane review manager [4, 5]. Only descriptive statistics and no statistical tests were used.

\section{Results}

\section{Literature search}

Figure 1 shows the flow of studies through the review process. As depicted in Fig. 1 the initial search resulted in 1913 potential studies. After removal of duplicates 1209 remained. After screening of the abstracts 1112 articles were excluded, leaving 97 papers left for full text assessment. After examination, 77 articles were excluded for various reasons as described in Fig. 1. Of the remaining studies there were still four studies of which surgical expertise was unclear and no reaction was obtained from the author; therefore these studies were finally excluded. The final selection included 16 papers matching the inclusion criteria.

\section{Outcome}

Included studies can be seen in Tables 2 and 3. Eight expert and non expert studies were included.

In total 3086 Lichtenstein procedures were performed, Within the expert group, CPIP varied between 11.7 and $6.9 \%$ declining over time. The four (out of 8) RCTs in the expert group identified as methodologically strongest showed an incidence of CPIP between 10.4 and $5.4 \%$ at 12-and 24-month interval.

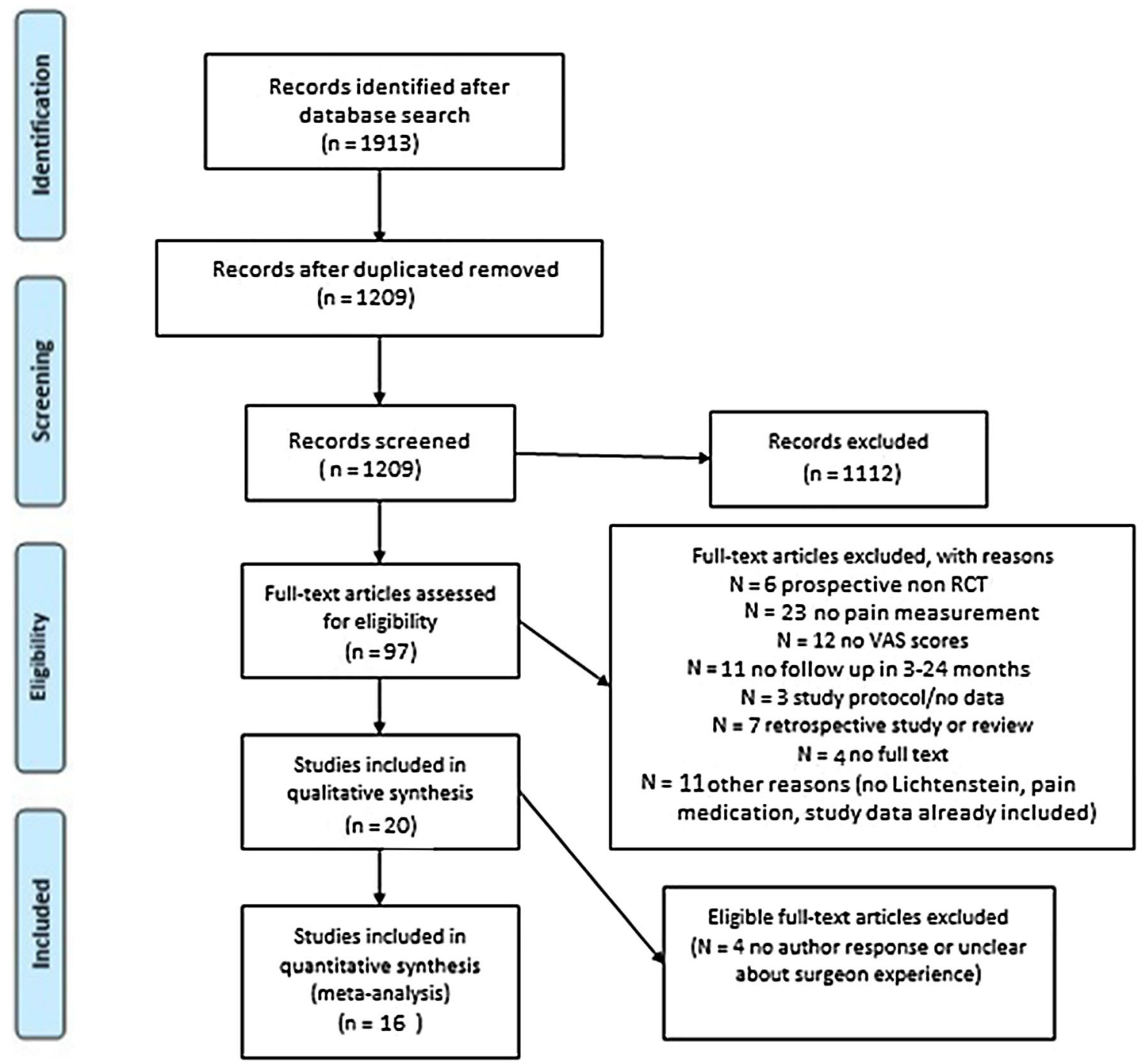

Fig. 1 Prisma flow diagram showing the flow of studies through the review process 
Table 2 Incidence of CPIP in expert group

\begin{tabular}{|c|c|c|c|c|c|c|}
\hline \multicolumn{3}{|l|}{ Expert studies } & \multirow[t]{2}{*}{3 months CPIP } & \multirow[t]{2}{*}{6 months CPIP } & \multirow[t]{2}{*}{12 months CPIP } & \multirow[t]{2}{*}{24 months CPIP } \\
\hline Author & No of & & & & & \\
\hline Jorgensen & 2012 & 334 & & & $29 / 329$ & \\
\hline Koch & 2008 & 317 & & & $17 / 317$ & \\
\hline Paajanen & 2011 & 302 & & & $51 / 286$ & \\
\hline Paajanen & 2012 & 312 & & & & $17 / 312$ \\
\hline Subtotal & & 1265 & & & $97 / 932$ & $17 / 312$ \\
\hline Subtotal (\%) & & & & & $10.4 \%$ & $5.4 \%$ \\
\hline Smietanski & 2011 & 199 & $39 / 199$ & $15 / 199$ & $14 / 199$ & \\
\hline Anadol & 2011 & 60 & & & & $11 / 27$ \\
\hline Dalenback & 2009 & 158 & $4 / 158$ & & $5 / 154$ & \\
\hline Demetrhashvili & 2014 & 198 & $22 / 198$ & & $15 / 198$ & $9 / 198$ \\
\hline Total & & 1880 & $65 / 555$ & $15 / 199$ & $131 / 1483$ & $37 / 537$ \\
\hline Total (\%) & & & $11.7 \%$ & $7.5 \%$ & $8.8 \%$ & $6.9 \%$ \\
\hline
\end{tabular}

In the non expert group the incidence was between 18.1 and $39.4 \%$. The incidence of CPIP in expert versus non expert studies is depicted in Fig. 2.

Overall, after 3 months 126 patients of the 767 patients (16.4\%) receiving follow-up complained of postoperative inguinal pain irrespective of surgical expertise. After 6 months there were 125 out of 378 patients (33\%), after 1 year 238 patients out of $1919(12.4 \%)$ patients and after 2 years there were still 88 patients out of $819(10.7 \%)$ complaining of CPIP. As well in the expert group as in the non-expert group the incidence of CPIP gradually deteriorated over time.

We assessed the methodological strength of RCTs based on a number of possible biases $[4,5]$. Based on this analysis we reported the incidence of CPIP in the four methodological strongest studies in the expert group separately (Table 2). In the non expert group the RCT of Koning et al. was methodologically strongest (Table 4).

All included studies quantified CPIP via VAS score. However, both measurement conditions and reporting differed amongst studies. The reporting of VAS scores is depicted in Table 5 below.

\section{Discussion}

With the introduction of prosthetic mesh CPIP has replaced recurrence as the major complication in inguinal hernia surgery. As experts in the field Amid and Chen are already reporting potential causes for CPIP for years and put the main focus on the role of the inguinal nerves, identification and preservation of the nerves when possible being their credo [6-8]. The course of the three inguinal nerves and potential variations are well known and it has been proved that identification and preservation are feasible in daily practice [9, 10]. However, it has also been shown in the past that a significant part of general surgeons performing inguinal hernia surgery is not familiar with the detailed nervous anatomy and the role that the nerves might play in CPIP [11]. This underlines the hypothesis that in inguinal hernia surgery performed by dedicated hernia surgeons the incidence of CPIP is reduced.

The suggestion that correlation between surgical expertise and CPIP exists is underlined by the results of this review. The incidence of CPIP after the Lichtenstein procedure is lower in the expert group compared to the non-expert group. Deysine et al. have shown this relation previously. Two groups undergoing hernioplasty were prospectively enrolled where one group was treated by a general surgeon and the second group was treated by a dedicated inguinal hernia surgeon. The aforementioned group showed significant better short- and long-term outcomes than the group treated by surgeons not dedicated to the field of inguinal hernia repair [12]. However, Cueto Rozon et al. compared the outcome (including CPIP) after the Lichtenstein procedure in three operating teams differing in expertise finding no difference in CPIP. In this series though, the experience level varied from intern to surgeon. Although experience was the main variable, no comparison was made between dedicated inguinal hernia surgeons and non-experts [13].

CPIP aside, surgical experience has been shown to reduce another serious complication of Lichtenstein procedure: recurrence of hernia [14]. Analysis of the Danish Hernia databasa, unique because of its complete follow-up, has shown that surgeons performing Lichtenstein procedures in private practice have lower recurrence rates than those operating in general hospitals. Performed under local anaesthesia, in private practice recurrence rates of direct hernias were $50 \%$ lower than those in general hospitals. 
Table 3 Incidence of CPIP in non expert group

\begin{tabular}{|c|c|c|c|c|c|c|}
\hline \multicolumn{3}{|l|}{ Non expert studies } & \multirow[t]{2}{*}{3 months CPIP } & \multirow[t]{2}{*}{6 months CPIP } & \multirow[t]{2}{*}{12 months CPIP } & \multirow[t]{2}{*}{24 months CPIP } \\
\hline Author & \multicolumn{2}{|c|}{ No of operations } & & & & \\
\hline Champault & 2007 & 237 & & & & \\
\hline Nienhuis & 2008 & 86 & $34 / 84$ & & & \\
\hline Sadowski & 2008 & 78 & $14 / 78$ & & & \\
\hline Langeveld & 2010 & 317 & & & $65 / 231$ & \\
\hline Koning & 2012 & 159 & & & $80 / 155$ & \\
\hline Nikkolo (1) & 2014 & 134 & & $54 / 134$ & & \\
\hline Nikkolo (2) & 2014 & 145 & & $56 / 145$ & & \\
\hline Chatzimavroudis & 2014 & 50 & $13 / 50$ & & $7 / 50$ & $3 / 50$ \\
\hline Total & & 1206 & $61 / 212$ & $110 / 279$ & $152 / 436$ & $51 / 282$ \\
\hline Total (\%) & & & $28.8 \%$ & $39.4 \%$ & $34.8 \%$ & $18.1 \%$ \\
\hline
\end{tabular}

Fig. 2 CPIP in expert versus non expert RCT's over time in percentage. The incidence is higher in the non expert group in all time intervals

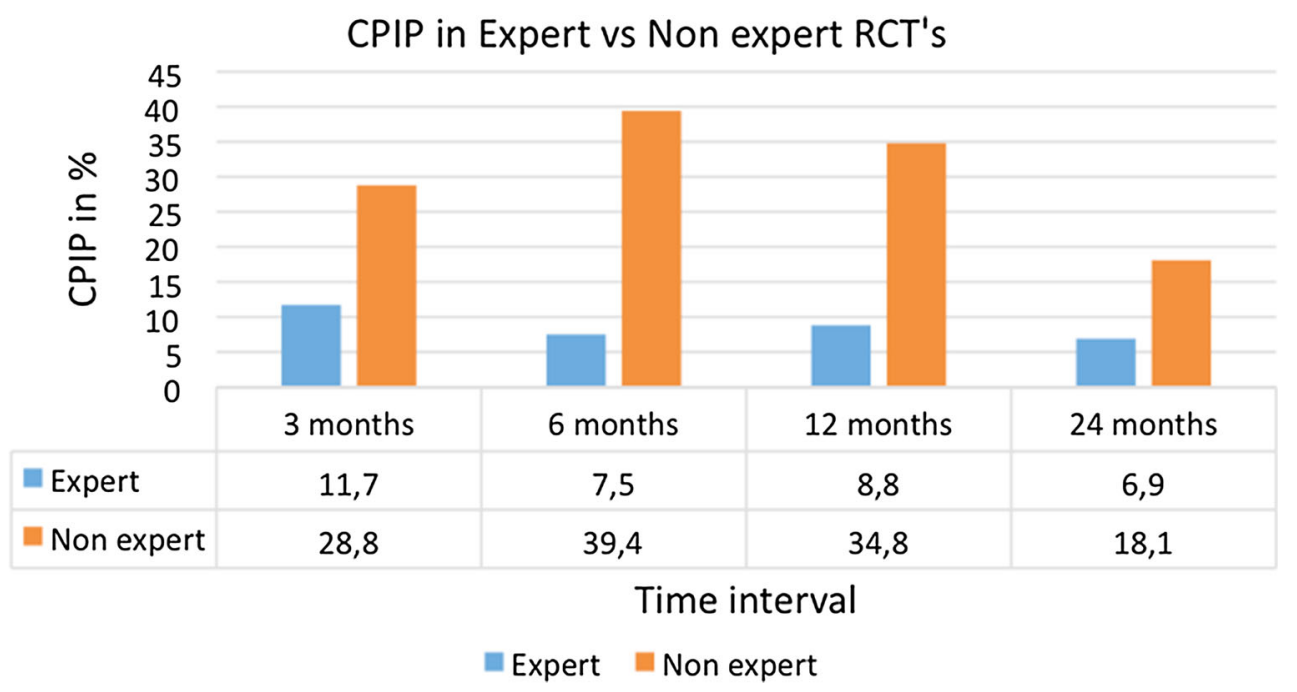

One may consider that the experience of these aforementioned "hernia experts" is a plausible explanation for these results [15, 16]. As a matter of fact, Kingsnorth et al. [17] found in a series of 183 Liechtenstein procedures performed under local anaesthesia that surgeon's experience was the single most important factor to predict recurrence rate, more so than type of hernia, chronic cough, heavy lifting or returning to work. If this is the case for recurrence rate after Lichtenstein, querying a similar learning curve for CPIP would be interesting at least.

Such a relation between CPIP and surgeon experience appears plausible for two reasons. First, the aforementioned analogy with surgical experience and recurrence rate was demonstrated in the same procedure. Second, other types of surgery also demonstrate improved outcomes after an initial learning curve. It is probable that CPIP could be a complication that diminishes in importance as the surgeon becomes more familiar with the anatomy and specific technique of the procedure. The Lichtenstein technique, as any other so-called surgical 'minor' operation, can be easily underestimated with regard to complexity. A stateof-the-art Lichtenstein procedure however is not that easy: a "nerveminded" approach with nervesparing intentions, described by Amid, demands skills and a good knowledge of the anatomy to prevent acute postoperative pain or CPIP. In this respect the authors want to nuance the guidelines of the European Hernia Society, which suggest that Lichtenstein repair by supervised residents or nonexperts is as good as that by experts. The evidence where this is based on is very weak, because the concerning literature is outdated and not or inadequately focusing on CPIP [13, 18].

This review has several limitations. The reporting on pain scores differed amongst studies. VAS scores were obtained for the whole study population or only the CPIP subgroup with regard to either rest, exercise or no defined condition at all. Therefore, a relevant comparison of pain qualification could not be made. CPIP was defined as (any) 
Table 4 Risk of bias summary: each risk of bias item for each included randomised controlled study [5]

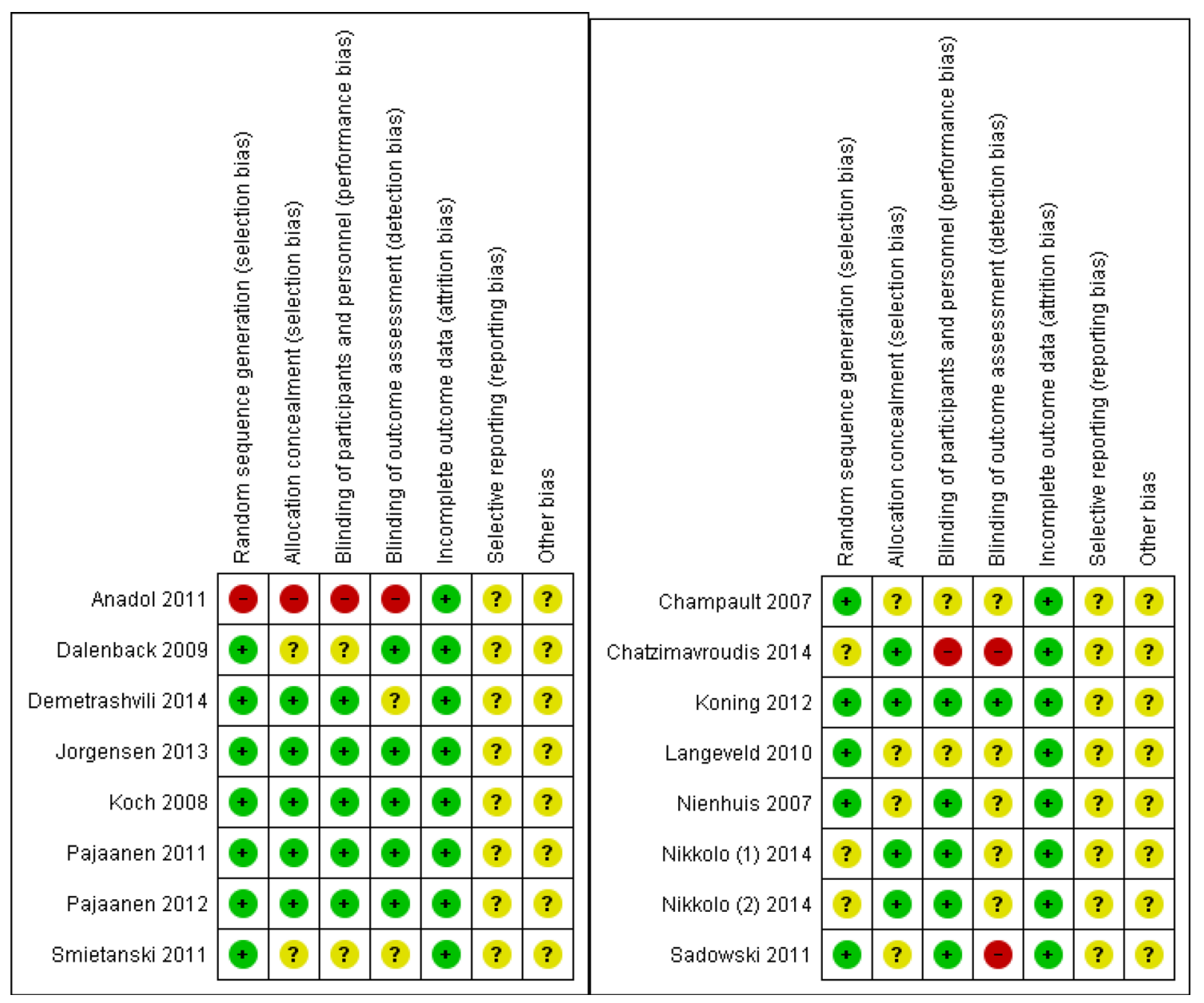

Left side reports on expert studies, on the right the non-expert studies

chronic inguinal pain after 3 months of the Lichtenstein procedure. In order to include all reports of CPIP we included the pain scores at the condition (rest/exercise) for which the incidence was highest, if reported. In ten studies this was not specified. We acknowledge the heterogeneity of reporting on CPIP although it is not unthinkable that the patient will report on inguinal pain for any condition when asked for groin pain at follow-up.

Although methodologically good studies were found, they were few and great heterogeneity between groups was observed. The variety in study designs led to anticipated limitations of this review. Foremost, there was no uniform research question between all included studies. Second, there is substantial heterogeneity between groups (inclusion criteria, use of meshes, glue, fibrin, stitches). These limitations could have biased the results either way and (thus) no statistical test could be performed to demonstrate significance.

This overview of the current and most recent literature suggests the existence of a positive correlation between expertise and outcomes in inguinal hernia surgery although no significant conclusion could be reached for CPIP. This review can be considered as a basis to investigate the role of expertise in CPIP in future prospective studies.

Open Access This article is distributed under the terms of the Creative Commons Attribution-NonCommercial 4.0 International License (http://creativecommons.org/licenses/by-nc/4.0/), which permits any noncommercial use, distribution, and reproduction in any medium, provided you give appropriate credit to the original author(s) and the source, provide a link to the Creative Commons license, and indicate if changes were made. 
Table 5 Reporting on VAS scores. Both measurement conditions and reporting differed amongst studies

\begin{tabular}{|c|c|c|c|}
\hline & VAS measurement & Measurement conditions & Note \\
\hline \multicolumn{4}{|c|}{ Reporting on VAS scores } \\
\hline \multicolumn{4}{|l|}{ Expert studies } \\
\hline Joreensen & 2012 VAS score for whole study population & Not specified & \\
\hline Koch & 2008 VAS score for whole study population & Rest and exercise & Not at 3- and 12-month interval \\
\hline Paajanen & 2011 VAS score for whole study population & Exercise & \\
\hline Paajanen & 2012 VAS score for whole study population & Rest & \\
\hline Smietanski & 2011 VAS score for whole study population & Not specified & \\
\hline Anadol & 2011 VAS score for CPIP subgroup & Not specified & \\
\hline Dalenback & 2009 VAS score for whole study population & Not specified & Not at 3- and 12-month interval \\
\hline Demetrashvili & 2014 VAS score for CPIP subgroup & Not specified & \\
\hline \multicolumn{4}{|l|}{ Non expert studies } \\
\hline Champault & 2007 VAS score for CPIP subgroup & Not specified & In gradations ( $<5$ vs. $>5$ ) \\
\hline Nienhuis & 2008 VAS score for whole study population & Not specified & Not at 3-month interval \\
\hline Sadowski & 2008 VAS score for CPIP subgroup & Not specified & In gradations \\
\hline Langeveld & 2010 VAS score for whole study population & Not specified & Not at 12 -month interval \\
\hline Koning & 2012 VAS score for whole study population & Rest and exercise & Not at 12 -month interval \\
\hline Nikkolo (1) & 2014 VAS score for CPIP subgroup & Rest and exercise & \\
\hline Nikkolo (2) & 2014 VAS score for CPIP subgroup & Rest and exercise & \\
\hline Chatzimavroudis & 2014 VAS score for CPIP subgroup & Not specified & \\
\hline
\end{tabular}

\section{Appendix: Included studies}

\section{Expert studies}

Anadol AZ, Akin M, Kurukahvecioglu O, Tezel E, Ersoy E. A prospective comparative study of the efficacy of conventional Lichtenstein versus self-adhesive mesh repair for inguinal hernia. Surg Today 2011 Nov;41(11): 1498-1503.

Dalenback J, Andersson C, Anesten B, Bjorck S, Eklund $\mathrm{S}$, Magnusson O, et al. Prolene Hernia System, Lichtenstein mesh and plug-and-patch for primary inguinal hernia repair: 3-year outcome of a prospective randomised controlled trial. The BOOP study: bi-layer and connector, onlay, and on-lay with plug for inguinal hernia repair. Hernia 2009 Apr;13(2):121-9; discussion 231.

Demetrashvili Z, Khutsishvili K, Pipia I, Kenchadze G, Ekaladze E. Standard polypropylene mesh vs lightweight mesh for Lichtenstein repair of primary inguinal hernia: A randomized controlled trial. Int $\mathbf{J}$ of Surg 2014;12: $1380-1384$.

Jorgensen LN, Sommer T, Assaadzadeh S, Strand L, Dorfelt A, Hensler M, et al. Randomized clinical trial of self-gripping mesh versus sutured mesh for Lichtenstein hernia repair. Br J Surg 2013 Mar;100(4):474-481.

Koch A, Bringman S, Myrelid P, Smeds S, Kald A. Randomized clinical trial of groin hernia repair with titanium-coated lightweight mesh compared with standard polypropylene mesh. Br J Surg 2008 Oct;95(10): 1226-1231.

Paajanen H, Kossi J, Silvasti S, Hulmi T, Hakala T. Randomized clinical trial of tissue glue versus absorbable sutures for mesh fixation in local anaesthetic Lichtenstein hernia repair. Br J Surg 2011 Sep;98(9):1245-1251.

Paajanen H, Ronka K, Laurema A. A single-surgeon randomized trial comparing three meshes in lichtenstein hernia repair: 2- and 5-year outcome of recurrences and chronic pain. Int J Surg 2013;11(1):81-84.

Smietanski M, Bury K, Smietanska IA, Owczuk R, Paradowski T, Polish Hernia Study Group. Five-year results of a randomised controlled multi-centre study comparing heavy-weight knitted versus low-weight, nonwoven polypropylene implants in Lichtenstein hernioplasty. Hernia 2011 Oct;15(5):495-501.

\section{Non expert studies}

Champault G, Bernard C, Rizk N, Polliand C. Inguinal hernia repair: the choice of prosthesis outweighs that of technique. Hernia 2007 Apr;11(2):125-128.

Chatzimavroudis G, Papaziogas B, Koutelikadis I, Galanis I, Atzamidis S, Christopoulos P, Doulias T, Atzamadis K, Makris J. Lichtenstein technique for inguinal hernia repair using polyproylene mesh fixed with sutures vs. self-fixating polyprolylene mesh: a prospective randomized comparative study. Hernia 2014;18:193-198 
Koning GG, Keus F, Koeslag L, Cheung CL, Avci M, van Laarhoven CJ, et al. Randomized clinical trial of chronic pain after the transinguinal preperitoneal technique compared with Lichtenstein's method for inguinal hernia repair. Br J Surg 2012 Oct;99(10):1365-1373.

Langeveld HR, van't Riet M, Weidema WF, Stassen LP, Steyerberg EW, Lange J, et al. Total extraperitoneal inguinal hernia repair compared with Lichtenstein (the LEVEL-Trial): a randomized controlled trial. Ann Surg 2010 May;251(5):819-824.

Nienhuijs S, Staal E, Keemers-Gels M, Rosman C, Strobbe L. Pain after open preperitoneal repair versus Lichtenstein repair: a randomized trial. World J Surg 2007 Sep;31(9):1751-7; discussion 1758-9.

Nikkolo C, Vaasna T, Murruste M, Seepter H, Kirsimagi U, Lepner U. Randomized clinical study evaluating the impact of mesh pore size on chronic pain after Lichtenstein hernioplasty. J of Surg Research 2014;191:311-317

Nikkolo C, Vaasna T, Murruste M, Seepter H, Suuman J, Tein A, Kirsimagi U, Lepner U. Single center, single blinded, randomized study of self gripping versus sutured mesh in open inguinal hernia repair. J of Surg Research 2015;194:77-82.

Sadowski B, Rodriguez J, Symmonds R, Roberts J, Song J, Rajab MH, et al. Comparison of polypropylene versus polyester mesh in the Lichtenstein hernia repair with respect to chronic pain and discomfort. Hernia 2011 Dec;15(6):643-654.

\section{References}

1. Archampong D, Borowski D, Wille-Jorgensen P, Iversen LH (2012) Workload and surgeon's specialty for outcome after colorectal cancer surgery. Cochrane Database Syst Rev 3:CD005391

2. Coupland VH, Lagergren J, Luchtenborg M, Jack RH, Allum W, Holmberg L et al (2013) Hospital volume, proportion resected and mortality from oesophageal and gastric cancer: a populationbased study in England, 2004-2008. Gut 62(7):961-966
3. Moher D, Liberati A, Tetzlaff J, Altman DG, PRISMA Group (2010) Preferred reporting items for systematic reviews and metaanalyses: the PRISMA statement. Int J Surg 8(5):336-341

4. The Nordic Cochrane Centre (2014) The Cochrane collaboration. Review Manager (RevMan) [Computer program]

5. Higgins JPT, Green S (2011) Cochrane handbook for systematic reviews of interventions. Version 5.1.0 ed.: The Cochrane Collaboration

6. Amid PK (2007) New understanding of the causes and surgical treatment of postherniorrhaphy inguinodynia and orchalgia. J Am Coll Surg 205:381-385

7. Amid PK (2004) Causes, prevention, and surgical treatment of postherniorrhaphy inguinodynia: tripl neurectomy with proximal end implantation. Hernia 8:343-349

8. Chen DC, Amid PK (2012) Questioning the favorable influence of nerve identification during open inguinal herniorrhaphy is misleading and may increases the risk of surgeons' liability. Hernia 16(5):579

9. Wijsmuller AR, Lange JFM, Kleinrensink GJ et al (2007) Nerveidentifying inguinal hernia repair: a surgical anatomical study. World J Surg 31(2):414-420

10. Lange JF, Wijsmuller AR (2009) Feasibility study of three-nerverecognizing Lichtenstein procedure for inguinal hernia. Br J Surg 96(10): 1210

11. Wijsmuller AR, Lange JFM, van Geldere D et al (2007) Surgical technique preventing chronic pain after Lichtenstein hernia repair; state of the art vs. daily practice in the Netherlands. Hernia 11(2):147-151

12. Deysine M, Grimson RC, Soroff HS (1991) Inguinal herniorrhaphy. Reduced morbidity by service standardization. Arch Surg 126(5):628-630

13. Cueto Rozon R, De Baerdemacker Y, Polliand C, Champault G (2006) Surgical training and inguinal hernia repair. Ann Chir 131(5):311-315

14. Nordin P, Haapaniemi S, van der Linden W, Nilsson E (2004) Choice of anesthesia and risk of reoperation for recurrence in groin hernia repair. Ann Surg 240(1):187-192

15. Sorensen LT, Friis E, Jorgensen T, Vennits B, Andersen BR, Rasmussen GI et al (2002) Smoking is a risk factor for recurrence of groin hernia. World J Surg 26(4):397-400

16. Kehlet H, Bay Nielsen M (2005) Anaesthetic practice for groin hernia repair-a nation-wide study in Denmark 1998-2003. Acta Anaesthesiol Scand 49(2):143-146

17. Kingsnorth AN, Britton BJ, Morris PJ (1981) Recurrent inguinal hernia after local anaesthetic repair. Br J Surg 68(4):273-275

18. Shulman AG, Amid PK, Lichtenstein IL (1995) A survey of nonexpert surgeons using the open tension-free mesh patch repair for primary inguinal hernias. Int Surg 80:35-36 